\title{
TRANSFORMS AND CONVOLUTIONS ON FUNCTION SPACE
}

\author{
Seung Jun Chang and Jae Gil Choi
}

\begin{abstract}
In this paper, for functionals of a generalized Brownian motion process, we show that the generalized Fourier-Feynman transform of the convolution product is a product of multiple transforms and that the conditional generalized Fourier-Feynman transform of the conditional convolution product is a product of multiple conditional transforms. This allows us to compute the (conditional) transform of the (conditional) convolution product without computing the (conditional) convolution product.
\end{abstract}

\section{Introduction}

For $f \in L^{2}\left(\mathbb{R}^{n}\right)$, let the Fourier transform of $f$ be given by

$$
\mathcal{F}(f)(u)=\int_{\mathbb{R}^{n}} e^{i u v} f(v) d m_{n}(v)
$$

and for $f, g \in L^{2}\left(\mathbb{R}^{n}\right)$, let the convolution of $f$ and $g$ be given by

$$
(f * g)(u)=\int_{\mathbb{R}^{n}} f(u-v) g(v) d m_{n}(v),
$$

where $d m_{n}(v)$ is the normalized Lebesgue measure $(2 \pi)^{-n / 2} d v$ on $\mathbb{R}^{n}$. The Fourier transform $\mathcal{F}$ satisfies the property of Parseval's relation in the form

$$
\int_{\mathbb{R}^{n}} f(v) g(v) d m_{n}(v)=\int_{\mathbb{R}^{n}} \mathcal{F}(f)(v) \mathcal{F}(g)(v) d m_{n}(v) .
$$

Furthermore $\mathcal{F}$ acts like a homomorphism with convolution $*$ and ordinary multiplication on $L^{2}\left(\mathbb{R}^{n}\right)$. More precisely, one can see for $f, g \in L^{2}\left(\mathbb{R}^{n}\right)$

$$
\mathcal{F}(f * g)=\mathcal{F}(f) \mathcal{F}(g)
$$

Received December 20, 2008.

2000 Mathematics Subject Classification. Primary 60J65, 28C20.

Key words and phrases. generalized Brownian motion process, generalized FourierFeynman transform, convolution product, conditional generalized Fourier-Feynman transform, conditional convolution product.

The present research was conducted by the research fund of Dankook University in 2008. 
and

$$
\mathcal{F}(f) * \mathcal{F}(g)=\mathcal{F}(f g) .
$$

Let $C_{0}[0, T]$ denote one-parameter Wiener space, that is, the space of realvalued continuous functions $x(t)$ on $[0, T]$ with $x(0)=0$. In [1] Brue introduced an $L_{1}$ analytic Fourier-Feynman transform(FFT) for functionals on Wiener space. There has been a tremendous amount of papers (including $L_{p}$-theory, $1 \leq p \leq 2$, see $[2,12])$ in the literature on the FFT theory. The FFT on Wiener space $C_{0}[0, T]$ is a transform of functionals which is somewhat analogous to the Fourier transform of functions.

In $[8,9,10,11,15]$, Huffman, Park, and Skoug defined an $L_{p}$ analytic FFT, a convolution product(CP), a conditional FFT(CFFT), and a conditional $\mathrm{CP}(\mathrm{CCP})$ for functionals on Wiener space and obtained various results involving and relating the FFT, the $\mathrm{CP}$, the CFFT and the CCP. In particular, they showed that the FFT of the CP is the product of transforms and that the conditional transform of the conditional convolution is the product of conditional transforms.

In this paper, we also study some relationships between a generalized FFT (GFFT) and a CP, and between a conditional GFFT(CGFFT) and a CCP on a very general function space $C_{a, b}[0, T]$ rather than on the Wiener space $C_{0}[0, T]$. The function space $C_{a, b}[0, T]$ induced by a generalized Brownian motion process was introduced by J. Yeh [16] and was used extensively by Chang and Chung [6]. But our results between the GFFT and the CP, and between the CGFFT and the CCP on function space are different from those in $[8,9,10,11,15]$. For example, the GFFT does not act like a homomorphism on the space of functionals on $C_{a, b}[0, T]$.

The Wiener process used in $[8,9,10]$ is stationary in time and is free of drift and the Gaussian process used in $[11,15]$ is nonstationary in time and free of drift. But the stochastic process used in this paper as well as in $[3,4,5,6,7,16]$, is nonstationary in time and is subject to a drift $a(t)$. However, when $a(t) \equiv 0$ and $b(t)=t$ on $[0, T]$, the general function space $C_{a, b}[0, T]$ reduces to the Wiener space $C_{0}[0, T]$.

\section{Definitions and preliminaries}

In this section we briefly list some of the preliminaries from $[3,4,7]$ that we need to establish our results in Sections 3 and 4 below; for more details see $[3,4,7]$.

Let $\left(C_{a, b}[0, T], \mathcal{B}\left(C_{a, b}[0, T]\right), \mu\right)$ denote the function space induced by a generalized Brownian motion process $Y$ determined by $a(t)$ and $b(t)$, where $\mathcal{B}\left(C_{a, b}[0, T]\right)$ is the Borel $\sigma$-algebra induced by sup-norm, see [16] and [17, Chapters 3 and 4]. We assume in this paper that $a(t)$ is an absolutely continuous real-valued function on $[0, T]$ with $a(0)=0, a^{\prime}(t) \in L^{2}[0, T]$, and $b(t)$ is a strictly increasing, continuously differentiable real-valued function with 
$b(0)=0$ and $b^{\prime}(t)>0$ for each $t \in[0, T]$. Then we can consider the coordinate process $X: D \times C_{a, b}[0, T] \rightarrow \mathbb{R}$ given by $X(t, x)=x(t)$ which is the continuous realization of $Y[17$, Theorem 14.2]. That is, for any $t \in[0, T]$ and $x \in C_{a, b}[0, T]$ we have

$$
X(t, x)=x(t) \sim N(a(t), b(t)) .
$$

A subset $B$ of $C_{a, b}[0, T]$ is said to be scale-invariant measurable(s.i.m.) provided $\rho B$ is $\mathcal{B}\left(C_{a, b}[0, T]\right)$-measurable for all $\rho>0$, and a scale-invariant measurable set $N$ is said to be a scale-invariant null set provided $\mu(\rho N)=0$ for all $\rho>0$. A property that holds except on a scale-invariant null set is said to hold scale-invariant almost everywhere(s-a.e.). If two functionals $F$ and $G$ defined on $C_{a, b}[0, T]$ are equal s-a.e., then we write $F \approx G$.

Let $L_{a, b}^{2}[0, T]$ be the set of functions on $[0, T]$ which are Lebesgue measurable and square integrable with respect to the Lebesgue-Stieltjes measures on $[0, T]$ induced by $a(\cdot)$ and $b(\cdot)$ : i.e.,

$$
L_{a, b}^{2}[0, T]=\left\{v: \int_{0}^{T} v^{2}(s) d b(s)<\infty \text { and } \int_{0}^{T} v^{2}(s) d|a|(s)<\infty\right\},
$$

where $|a|(\cdot)$ is the total variation function of $a(\cdot)$. Then $L_{a, b}^{2}[0, T]$ is a separable Hilbert space with inner product defined by

$$
(u, v)_{a, b}=\int_{0}^{T} u(t) v(t) d[b(t)+|a|(t)] .
$$

In addition, for each $v \in L_{a, b}^{2}[0, T]$, the Paley-Wiener-Zygmund(PWZ) stochastic integral $\langle v, x\rangle$ exists for $\mu$-a.e. $x \in C_{a, b}[0, T]$. If $v$ is of bounded variation on $[0, T]$, then the PWZ stochastic integral $\langle v, x\rangle$ equals the Riemann-Stieltjes integral $\int_{0}^{T} v(t) d x(t)$ for s-a.e. $x \in C_{a, b}[0, T]$. For more details, see [7].

Remark 2.1. For each $v \in L_{a, b}^{2}[0, T]$, the PWZ stochastic integral $\langle v, x\rangle$ is a Gaussian random variable on $C_{a, b}[0, T]$ with mean $\int_{0}^{T} v(s) d a(s)$ and variance $\int_{0}^{T} v^{2}(s) d b(s)$. Note that for all $u, v \in L_{a, b}^{2}[0, T]$,

$$
\begin{aligned}
& \int_{C_{a, b}[0, T]}\langle u, x\rangle\langle v, x\rangle d \mu(x) \\
= & \int_{0}^{T} u(s) v(s) d b(s)+\int_{0}^{T} u(s) d a(s) \int_{0}^{T} v(s) d a(s) .
\end{aligned}
$$

Hence we see that for all $u, v \in L_{a, b}^{2}[0, T], \int_{0}^{T} v(s) u(s) d b(s)=0$ if and only if $\langle u, x\rangle$ and $\langle v, x\rangle$ are independent random variables.

Let

$$
C_{a, b}^{\prime}[0, T]=\left\{w \in C_{a, b}[0, T]: w(t)=\int_{0}^{t} z(s) d b(s) \text { for some } z \in L_{a, b}^{2}[0, T]\right\}
$$


For $w \in C_{a, b}^{\prime}[0, T]$, with $w(t)=\int_{0}^{t} z(s) d b(s)$ for $t \in[0, T]$, let $D_{t}: C_{a, b}^{\prime}[0, T] \rightarrow$ $L_{a, b}^{2}[0, T]$ be defined by the formula

$$
D_{t} w=z(t)=\frac{w^{\prime}(t)}{b^{\prime}(t)} .
$$

Then $C_{a, b}^{\prime} \equiv C_{a, b}^{\prime}[0, T]$ with inner product

$$
\left(w_{1}, w_{2}\right)_{C_{a, b}^{\prime}}=\int_{0}^{T} D_{t} w_{1} D_{t} w_{2} d b(t)=\int_{0}^{T} z_{1}(t) z_{2}(t) d b(t)
$$

is a separable Hilbert space. Furthermore, $\left(C_{a, b}^{\prime}[0, T], C_{a, b}[0, T], \mu\right)$ is an example of abstract Wiener space. For more details, see [13].

Note that the two separable Hilbert spaces $L_{a, b}^{2}[0, T]$ and $C_{a, b}^{\prime}[0, T]$ are isomorphic under the linear operator given by equation (2.2), but they are not isometric.

For $w \in C_{a, b}^{\prime}[0, T]$, with $w(t)=\int_{0}^{t} z(s) d b(s)$ for $t \in[0, T]$, we will use the notation $(w, x)^{\sim}$ instead of $\langle z, x\rangle=\left\langle D_{t} w, x\right\rangle$. Then we have the following assertions.

(1) For each $w \in C_{a, b}^{\prime}[0, T]$, the random variable $x \mapsto(w, x)^{\sim}$ is Gaussian with mean $(w, a)_{C_{a, b}^{\prime}}$ and variance $\|w\|_{C_{a, b}^{\prime}}^{2}$.

(2) $(w, \alpha x)^{\sim}=\alpha(w, x)^{\sim}=(\alpha w, x)^{\sim}$ for any real number $\alpha, w \in C_{a, b}^{\prime}[0, T]$ and $x \in C_{a, b}[0, T]$.

(3) If $\left\{w_{1}, w_{2}, \ldots, w_{n}\right\}$ is an orthonormal set in $C_{a, b}^{\prime}[0, T]$, then the random variables $\left(w_{i}, x\right)^{\sim}$ 's are independent.

(4) Let $g$ be an element of $C_{a, b}^{\prime}[0, T]$ with $g(t)=\int_{0}^{t} \alpha(s) d b(s)$ for some $\alpha \in L_{a, b}^{2}[0, T]$. Then

$$
(w, g)^{\sim}=\int_{0}^{T} D_{t} w d g(t)=\int_{0}^{T} z(t) \alpha(t) d b(t)=(w, g)_{C_{a, b}^{\prime}} .
$$

We denote the function space integral of a $\mathcal{B}\left(C_{a, b}[0, T]\right)$-measurable functional $F$ by

whenever the integral exists.

$$
E[F]=\int_{C_{a, b}[0, T]} F(x) d \mu(x)
$$

Remark 2.2. (1) For $s, t \in[0, T]$ with $s<t$, we have the facts that

$$
E[x(t)]=a(t) \quad \text { and } \quad E[x(s) x(t)]=a(s) a(t)+b(s) .
$$

(2) Let $w \in C_{a, b}^{\prime}[0, T]$. Then

$$
\begin{aligned}
E\left[(w, x)^{\sim}\right] & =E\left[\left\langle D_{t} w, x\right\rangle\right]=\int_{0}^{T} D_{t} w d a(t) \\
& =\int_{0}^{T} D_{t} w D_{t} a d b(t)=(w, a)_{C_{a, b}^{\prime}} .
\end{aligned}
$$


(3) Let $g$ be an element of $C_{a, b}^{\prime}[0, T]$ with $g(t)=\int_{0}^{t} \alpha(s) d b(s)$ for some $\alpha \in L_{a, b}^{2}[0, T]$. Then using equation (2.1) we have that for each $t \in[0, T]$

$$
\begin{aligned}
& E\left[x(t)(g, x)^{\sim}\right]=E\left[\left\langle\chi_{[0, t]}, x\right\rangle\langle\alpha, x\rangle\right] \\
= & \int_{0}^{T} \chi_{[0, t]}(s) d a(s) \int_{0}^{T} \alpha(s) d a(s)+\int_{0}^{T} \chi_{[0, t]}(s) \alpha(s) d b(s) \\
= & a(t)(g, a)_{C_{a, b}^{\prime}}+g(t) .
\end{aligned}
$$

\section{Transform and convolution on function space}

In this section we define the GFFT and the CP on function space $C_{a, b}[0, T]$. We then investigate a relationship between the GFFT and the CP.

Throughout this paper we will assume that each functional $F$ (or $G$ ) we consider satisfies the conditions:

$$
F: C_{a, b}[0, T] \rightarrow \mathbb{C} \text { is s.i.m. and s-a.e. defined, }
$$

and

$$
E_{x}[|F(\rho x)|]<\infty \text { for each } \rho>0 .
$$

First we state the definition of the GFFT.

Definition 3.1. Let $\mathbb{C}_{+}=\{\lambda \in \mathbb{C}: \operatorname{Re}(\lambda)>0\}$ and let $\tilde{\mathbb{C}}_{+}=\{\lambda \in \mathbb{C}: \lambda \neq$ 0 and $\operatorname{Re}(\lambda) \geq 0\}$. Let $F$ satisfy conditions (3.1) and (3.2) above. If there exists a function $J^{*}(\lambda)$ analytic in $\mathbb{C}_{+}$such that $J^{*}(\lambda)=E_{x}\left[F\left(\lambda^{-1 / 2} x\right)\right]$ for all $\lambda>0$, then $J^{*}(\lambda)$ is defined to be the analytic function space integral of $F$ over $C_{a, b}[0, T]$ with parameter $\lambda$, and for $\lambda \in \mathbb{C}_{+}$we write

$$
E^{\mathrm{an}_{\lambda}}[F] \equiv E_{x}^{\mathrm{an}_{\lambda}}[F(x)]=J^{*}(\lambda) .
$$

For $\lambda \in \mathbb{C}_{+}$and $y \in C_{a, b}[0, T]$, let

$$
T_{\lambda}(F)(y)=E_{x}^{\mathrm{an}_{\lambda}}[F(y+x)] .
$$

For $p \in(1,2]$ we define the $L_{p}$ analytic $\operatorname{GFFT}, T_{q}^{(p)}(F)$ of $F$, by the formula $\left(\lambda \in \mathbb{C}_{+}\right)$,

$$
T_{q}^{(p)}(F)(y)=\text { l.i.m. } \lambda \rightarrow-i q T_{\lambda}(F)(y)
$$

if it exists; i.e., for each $\rho>0$,

$$
\lim _{\lambda \rightarrow-i q} \int_{C_{a, b}[0, T]}\left|T_{\lambda}(F)(\rho y)-T_{q}^{(p)}(F)(\rho y)\right|^{p^{\prime}} d \mu(y)=0,
$$

where $1 / p+1 / p^{\prime}=1$. We define the $L_{1}$ analytic $\operatorname{GFFT}, T_{q}^{(1)}(F)$ of $F$, by the formula $\left(\lambda \in \mathbb{C}_{+}\right)$

$$
T_{q}^{(1)}(F)(y)=\lim _{\lambda \rightarrow-i q} T_{\lambda}(F)(y)
$$

if it exists. 
We note that for $1 \leq p \leq 2, T_{q}^{(p)}(F)$ is defined only s-a.e.. We also note that if $T_{q}^{(p)}(F)$ exists and if $F \approx G$, then $T_{q}^{(p)}(G)$ exists and $T_{q}^{(p)}(G) \approx T_{q}^{(p)}(F)$.

Next we give the definition of the CP on function space $C_{a, b}[0, T]$.

Definition 3.2. Let $F$ and $G$ be functionals on $C_{a, b}[0, T]$. For $\lambda \in \tilde{\mathbb{C}}_{+}$, we define their $\mathrm{CP}(F * G)_{\lambda}$ (if it exists) by

$$
(F * G)_{\lambda}(y)= \begin{cases}E_{x}^{\operatorname{an}_{\lambda}}\left[F\left(\frac{y+x}{\sqrt{2}}\right) G\left(\frac{y-x}{\sqrt{2}}\right)\right], & \lambda \in \mathbb{C}_{+} \\ E_{x}^{\operatorname{anf}_{q}}\left[F\left(\frac{y+x}{\sqrt{2}}\right) G\left(\frac{y-x}{\sqrt{2}}\right)\right], & \lambda=-i q, q \in \mathbb{R}, q \neq 0 .\end{cases}
$$

Remark 3.3. (1) When $\lambda=-i q$, we denote $(F * G)_{\lambda}$ by $(F * G)_{q}$.

(2) Our convolution is not commutative.

To obtain our main result, we need the following lemma.

Lemma 3.4. Let $Y_{1}, Y_{2}:[0, T] \times C_{a, b}[0, T] \times C_{a, b}[0, T] \rightarrow \mathbb{R}$ be given by

$$
Y_{1}\left(t ; x_{1}, x_{2}\right)=\frac{x_{1}+x_{2}}{\sqrt{2}} \quad \text { and } \quad Y_{2}\left(t ; x_{1}, x_{2}\right)=\frac{x_{1}-x_{2}}{\sqrt{2}}
$$

Then $\left\{Y_{1}: t \in[0, T]\right\}$ and $\left\{Y_{2}: t \in[0, T]\right\}$ are independent processes.

Proof. Since the processes are Gaussian, it suffices to show that for every $t, s \in$ $[0, T]$,

$$
\begin{aligned}
& E_{x_{1}}\left[E_{x_{2}}\left[Y_{1}\left(s ; x_{1}, x_{2}\right) Y_{2}\left(t ; x_{1}, x_{2}\right)\right]\right] \\
= & E_{x_{1}}\left[E_{x_{2}}\left[Y_{1}\left(s ; x_{1}, x_{2}\right)\right]\right] E_{x_{1}}\left[E_{x_{2}}\left[Y_{2}\left(t ; x_{1}, x_{2}\right)\right]\right] .
\end{aligned}
$$

By equation (2.3), we can obtain that

$$
E_{x_{k}}\left[x_{k}(t)\right]=a(t) \text { and } E_{x_{k}}\left[x_{k}(s) x_{k}(t)\right]=\min \{b(s), b(t)\}+a(t) a(s)
$$

for $k=1,2$. Using these and direct calculations, we obtain the desired result.

We are now ready to establish one of our main results. In next theorem, we show that the GFFT of the CP is the product of multiple GFFTs.

Theorem 3.5. Let $F$ and $G$ be functionals on $C_{a, b}[0, T]$. Assume that

$$
T_{2 q}^{(p)}\left(T_{2 q}^{(p)}(F)\right), T_{2 q}^{(p)}\left(T_{2 q}^{(p)}(G(-\cdot))(-\cdot)\right)
$$

and $T_{q}^{(p)}\left((F * G)_{q}\right)$ all exist. Then

$$
\begin{aligned}
& T_{q}^{(p)}\left((F * G)_{q}\right)(y) \\
= & T_{2 q}^{(p)}\left(T_{2 q}^{(p)}(F)\right)\left(\frac{y}{\sqrt{2}}\right) T_{2 q}^{(p)}\left(T_{2 q}^{(p)}(G(-\cdot))(-\cdot)\right)\left(\frac{y}{\sqrt{2}}\right)
\end{aligned}
$$

for s-a.e. $y \in C_{a, b}[0, T]$. 
Proof. In view of the definition of the GFFT and the CP, it will suffice to show that

$$
T_{\lambda}\left((F * G)_{\lambda}\right)(y)=T_{2 \lambda}\left(T_{2 \lambda}(F)\right)(y / \sqrt{2}) T_{2 \lambda}\left(T_{2 \lambda}(G(-\cdot))(-\cdot)\right)(y / \sqrt{2})
$$

for $\lambda>0$. But for all $\lambda>0$,

$$
\begin{aligned}
& T_{\lambda}\left((F * G)_{\lambda}\right)(y) \\
= & E_{x_{1}}\left[(F * G)_{\lambda}\left(y+\lambda^{-1 / 2} x_{1}\right)\right] \\
= & E_{x_{1}}\left[E_{x_{2}}\left[F\left(\frac{y+\lambda^{-1 / 2} x_{1}+\lambda^{-1 / 2} x_{2}}{\sqrt{2}}\right) G\left(\frac{y+\lambda^{-1 / 2} x_{1}-\lambda^{-1 / 2} x_{2}}{\sqrt{2}}\right)\right]\right] .
\end{aligned}
$$

But by Lemma 3.4, we have that

$$
\begin{aligned}
& T_{\lambda}\left((F * G)_{\lambda}\right)(y) \\
= & E_{x_{1}}\left[E_{x_{2}}\left[F\left(\frac{y}{\sqrt{2}}+\frac{x_{1}}{\sqrt{2 \lambda}}+\frac{x_{2}}{\sqrt{2 \lambda}}\right)\right]\right] \\
& \times E_{x_{1}}\left[E_{x_{2}}\left[G\left(-\left[-\left(\frac{y}{\sqrt{2}}+\frac{x_{1}}{\sqrt{2 \lambda}}\right)+\frac{x_{2}}{\sqrt{2 \lambda}}\right]\right)\right]\right] \\
= & E_{x_{1}}\left[T_{2 \lambda}(F)\left(\frac{y}{\sqrt{2}}+\frac{x_{1}}{\sqrt{2 \lambda}}\right)\right] E_{x_{1}}\left[T_{2 \lambda}(G(-\cdot))\left(-\left(\frac{y}{\sqrt{2}}+\frac{x_{1}}{\sqrt{2 \lambda}}\right)\right)\right] \\
= & T_{2 \lambda}\left(T_{2 \lambda}(F)\right)\left(\frac{y}{\sqrt{2}}\right) T_{2 \lambda}\left(T_{2 \lambda}(G(-\cdot))(-\cdot)\right)\left(\frac{y}{\sqrt{2}}\right)
\end{aligned}
$$

which concludes the proof of Theorem 3.5.

Remark 3.6. Formula (3.3) is useful in that it permits one to calculate $T_{q}^{(p)}((F *$ $\left.G)_{q}\right)$ without actually calculating $(F * G)_{q}$.

\section{Conditional transform and conditional convolution}

In this section we define the conditional function space integral of $F$ given $X$ as the conditional expectation $E(F \mid X)$. We then define the conditional generalized Feynman integral, the $\operatorname{CGFFT} T_{q}^{(p)}(F \mid X)$ and the CCP $\left[(F * G)_{q} \mid X\right]$ on function space $C_{a, b}[0, T]$.

Throughout this section we will always condition by the function $X: C_{a, b}[0, T]$ $\rightarrow \mathbb{R}^{n}$

$$
X(x)=\left(\left(g_{1}, x\right)^{\sim}, \ldots,\left(g_{n}, x\right)^{\sim}\right),
$$

where $\left\{g_{1}, \ldots, g_{n}\right\}$ is an orthonormal set in $C_{a, b}^{\prime}[0, T]$.

In [14], Park and Skoug obtained a simple formula for expressing conditional Wiener integrals with a vector-valued conditioning function in terms of ordinary Wiener integrals, and then used the formula to derive the Kac-Feynman integral equation for time dependent potential function. In [15], they defined 
the concepts of a CFFT and a CCP on Wiener space using their simple formula, and studied several relationships between them. In $[14,15]$ the authors used the conditioning function $X: C_{0}[0, T] \rightarrow \mathbb{R}^{n}$ defined by

$$
X(x)=\left(x\left(t_{1}\right), \ldots, x\left(t_{n}\right)\right), 0=t_{0}<t_{1}<\cdots<t_{n}=T .
$$

We note that the conditioning function $X$ given by equation (4.2) is the special case of $X$ given by (4.1) with $b(t)=t$ and

$$
g_{j}(t)=\left[b\left(t_{j}\right)-b\left(t_{j-1}\right)\right]^{-1 / 2} \int_{0}^{t} \chi_{\left[t_{j-1}, t_{j}\right]}(s) d b(s)
$$

on $[0, T]$ for $j=1, \ldots, n$.

Let $X$ be a $\mathbb{R}^{n}$-valued function on $C_{a, b}[0, T]$ whose probability distribution $\mu_{X}$ is absolutely continuous with respect to Lebesgue measure on $\mathbb{R}^{n}$. Let $F: C_{a, b}[0, T] \rightarrow \mathbb{C}$ be $\mu$-integrable. Then the conditional integral of $F$ given $X$, denoted by $E(F \mid X)(\vec{\eta})$, is a Lebesgue measurable function of $\vec{\eta}$, unique up to null sets in $\mathbb{R}^{n}$, satisfying the equation

$$
\int_{X^{-1}(B)} F(x) d \mu(x)=\int_{B} E(F \mid X)(\vec{\eta}) d \mu_{X}(\vec{\eta})
$$

for all Borel sets $B$ in $\mathbb{R}^{n}$.

Definition 4.1. Let $F: C_{a, b}[0, T] \rightarrow \mathbb{C}$ satisfy conditions (3.1) and (3.2) and let $X: C_{a, b}[0, T] \rightarrow \mathbb{R}^{n}$ be given by (4.1). For $\lambda>0$ and $\vec{\eta} \in \mathbb{R}^{n}$, let

$$
J_{\lambda}(\vec{\eta})=E\left(F\left(\lambda^{-1 / 2} \cdot\right) \mid X\left(\lambda^{-1 / 2} \cdot\right)\right)(\vec{\eta})
$$

denote the conditional function space integral of $F\left(\lambda^{-1 / 2} \cdot\right)$ given $X\left(\lambda^{-1 / 2} \cdot\right)$. If for a.e. $\vec{\eta} \in \mathbb{R}^{n}$, there exists a function $J_{\lambda}^{*}(\vec{\eta})$ analytic in $\lambda$ on $\mathbb{C}_{+}$such that $J_{\lambda}^{*}(\vec{\eta})=J_{\lambda}(\vec{\eta})$ for all $\lambda>0$, then $J_{\lambda}^{*}(\cdot)$ is defined to be the conditional analytic function space integral of $F$ given $X$ with parameter $\lambda$ and for $\lambda \in \mathbb{C}_{+}$we write

$$
E^{\operatorname{an}_{\lambda}}(F \mid X)(\vec{\eta})=J_{\lambda}^{*}(\vec{\eta}) .
$$

If for fixed real $q \neq 0$, the limit

$$
\lim _{\lambda \rightarrow-i q} E^{\mathrm{an}_{\lambda}}(F \mid X)(\vec{\eta})
$$

exists for a.e. $\vec{\eta} \in \mathbb{R}^{n}$, where $\lambda \rightarrow-i q$ through values in $\mathbb{C}_{+}$, we denote the value of this limit by $E^{\operatorname{anf}_{q}}(F \mid X)(\vec{\eta})$ and we call it the conditional generalized analytic Feynman integral of $F$ given $X$ with parameter $q$.

The following theorem is useful to define the CGFFT and the CCP over $C_{a, b}[0, T][5]$.

Theorem 4.2. Let $F \in L^{1}\left(C_{a, b}[0, T]\right)$ and let $X$ be given by equation (4.1). Then

$$
E(F \mid X)(\vec{\eta})=E\left[F\left(x-\sum_{j=1}^{n}\left(g_{j}, x\right)^{\sim} g_{j}+\sum_{j=1}^{n} \eta_{j} g_{j}\right)\right]
$$


In view of Theorem 4.2 we can define the CGFFT and the CCP of functionals on function space $C_{a, b}[0, T]$.

Definition 4.3. For $\lambda \in \mathbb{C}_{+}$and $y \in C_{a, b}[0, T]$, let $T_{\lambda}(F \mid X)(y, \vec{\eta})$ denote the conditional analytic function space integral of $F(y+\cdot)$ given $X(\cdot)$; that is to say

$$
\begin{aligned}
T_{\lambda}(F \mid X)(y, \vec{\eta}) & =E^{\mathrm{an}_{\lambda}}(F(y+\cdot) \mid X)(\vec{\eta}) \\
& =E_{x}^{\mathrm{an}_{\lambda}}\left[F\left(y+x-\sum_{j=1}^{n}\left(g_{j}, x\right)^{\sim} g_{j}+\sum_{j=1}^{n} \eta_{j} g_{j}\right)\right] .
\end{aligned}
$$

Then for $p \in[1,2]$ we define the CGFFT of $F$ given $X$ by the formula $\left(\lambda \in \mathbb{C}_{+}\right)$,

$$
T_{q}^{(p)}(F \mid X)(y, \vec{\eta})=\left\{\begin{array}{lc}
\text { l.i.m. }{ }_{\lambda \rightarrow-i q} T_{\lambda}(F \mid X)(y, \vec{\eta}), & 1<p \leq 2 \\
\lim _{\lambda \rightarrow-i q} T_{\lambda}(F \mid X)(y, \vec{\eta}), & p=1
\end{array}\right.
$$

if it exists. Note that in the case $p=1$,

$$
T_{q}^{(1)}(F \mid X)(y, \vec{\eta})=E_{x}^{\operatorname{anf}_{q}}\left[F\left(y+x-\sum_{j=1}^{n}\left(g_{j}, x\right)^{\sim} g_{j}+\sum_{j=1}^{n} \eta_{j} g_{j}\right)\right] .
$$

And we define the $\operatorname{CCP}\left[(F * G)_{\lambda} \mid X\right](y, \vec{\eta})$ (if it exists) by the formula

$$
\begin{aligned}
& {\left[(F * G)_{\lambda} \mid X\right](y, \vec{\eta})} \\
& = \begin{cases}E^{\operatorname{an}_{\lambda}}\left(F\left(\frac{y+\cdot}{\sqrt{2}}\right) G\left(\frac{y-\cdot}{\sqrt{2}}\right) \mid X\right)(\vec{\eta}), & \lambda \in \mathbb{C}_{+} \\
E^{\operatorname{anf}_{q}}\left(F\left(\frac{y+\cdot}{\sqrt{2}}\right) G\left(\frac{y-\cdot}{\sqrt{2}}\right) \mid X\right)(\vec{\eta}), & \lambda=-i q, q \in \mathbb{R}, q \neq 0\end{cases} \\
& \left\{\begin{array}{c}
E_{x}^{\operatorname{an}_{\lambda}}\left[F\left(\frac{y+x}{\sqrt{2}}-\sum_{j=1}^{n} \frac{\left(g_{j}, x\right)^{\sim}}{\sqrt{2}} g_{j}+\sum_{j=1}^{n} \frac{\eta_{j}}{\sqrt{2}} g_{j}\right)\right. \\
\left.\times G\left(\frac{y-x}{\sqrt{2}}+\sum_{j=1}^{n} \frac{\left(g_{j}, x\right)^{\sim}}{\sqrt{2}} g_{j}-\sum_{j=1}^{n} \frac{\eta_{j}}{\sqrt{2}} g_{j}\right)\right], \quad \lambda \in \mathbb{C}_{+} \\
E_{x}^{\operatorname{anf}_{q}}\left[F\left(\frac{y+x}{\sqrt{2}}-\sum_{j=1}^{n} \frac{\left(g_{j}, x\right)^{\sim}}{\sqrt{2}} g_{j}+\sum_{j=1}^{n} \frac{\eta_{j}}{\sqrt{2}} g_{j}\right)\right. \\
\left.\times G\left(\frac{y-x}{\sqrt{2}}+\sum_{j=1}^{n} \frac{\left(g_{j}, x\right)^{\sim}}{\sqrt{2}} g_{j}-\sum_{j=1}^{n} \frac{\eta_{j}}{\sqrt{2}} g_{j}\right)\right], \quad \lambda=-i q, q \in \mathbb{R}, q \neq 0 .
\end{array}\right.
\end{aligned}
$$

Again if $\lambda=-i q$, we will denote $\left[(F * G)_{\lambda} \mid X\right](y, \vec{\eta})$ by $\left[(F * G)_{q} \mid X\right](y, \vec{\eta})$.

Lemma 4.4. Let $\left\{g_{1}, \ldots, g_{n}\right\}$ be an orthonormal set in $C_{a, b}^{\prime}[0, T]$. Let $Z_{1}, Z_{2}$ : $[0, T] \times C_{a, b}[0, T] \times C_{a, b}[0, T] \rightarrow \mathbb{R}$ be given by

$$
Z_{1}\left(t ; x_{1}, x_{2}\right)=2^{-1 / 2}\left(x_{1}(t)-\sum_{j=1}^{n}\left(g_{j}, x_{1}\right)^{\sim} g_{j}(t)+x_{2}(t)-\sum_{j=1}^{n}\left(g_{j}, x_{2}\right)^{\sim} g_{j}(t)\right)
$$

and

$$
Z_{2}\left(t ; x_{1}, x_{2}\right)=2^{-1 / 2}\left(x_{1}(t)-\sum_{j=1}^{n}\left(g_{j}, x_{1}\right)^{\sim} g_{j}(t)-x_{2}(t)+\sum_{j=1}^{n}\left(g_{j}, x_{2}\right)^{\sim} g_{j}(t)\right) .
$$


Then $\left\{Z_{1}: t \in[0, T]\right\}$ and $\left\{Z_{2}: t \in[0, T]\right\}$ are independent processes.

Proof. Using equations (2.1) through (2.5) and direct calculations, we can prove that for $s, t \in[0, T]$

$$
\begin{aligned}
& E_{x_{1}}\left[E_{x_{2}}\left[Z_{1}\left(s ; x_{1}, x_{2}\right) Z_{2}\left(t ; x_{1}, x_{2}\right)\right]\right] \\
= & E_{x_{1}}\left[E_{x_{2}}\left[Z_{1}\left(s ; x_{1}, x_{2}\right)\right]\right] E_{x_{1}}\left[E_{x_{2}}\left[Z_{2}\left(t ; x_{1}, x_{2}\right)\right]\right] .
\end{aligned}
$$

In Theorem 4.5 below, we show that the CGFFT of the CCP is the product of multiple CGFFTs.

Theorem 4.5. Let $X$ be given by equation (4.1). Let $F$ and $G$ be functionals on $C_{a, b}[0, T]$. Assume that

and

$$
\begin{gathered}
T_{2 q}^{(p)}\left(T_{2 q}^{(p)}(F \mid X)\left(\cdot, \vec{\eta}_{2}\right) \mid X\right)\left(y, \vec{\eta}_{1}\right), \\
T_{2 q}^{(p)}\left(T_{2 q}^{(p)}(G(-\cdot) \mid X)\left(-\cdot, \vec{\eta}_{2}\right) \mid X\right)\left(y, \vec{\eta}_{1}\right)
\end{gathered}
$$

all exist. Then

$$
T_{q}^{(p)}\left(\left[(F * G)_{q} \mid X\right]\left(\cdot, \vec{\eta}_{1}\right) \mid X\right)\left(y, \vec{\eta}_{2}\right)
$$

$$
\begin{aligned}
& T_{q}^{(p)}\left(\left[(F * G)_{q} \mid X\right]\left(\cdot, \vec{\eta}_{1}\right) \mid X\right)\left(y, \vec{\eta}_{2}\right) \\
= & T_{2 q}^{(p)}\left(T_{2 q}^{(p)}(F \mid X)\left(\cdot, \frac{\vec{\eta}_{2}}{\sqrt{2}}\right) \mid X\right)\left(\frac{y}{\sqrt{2}}, \frac{\vec{\eta}_{1}}{\sqrt{2}}\right) \\
& \times T_{2 q}^{(p)}\left(T_{2 q}^{(p)}(G(-\cdot) \mid X)\left(-\cdot,-\frac{\vec{\eta}_{2}}{\sqrt{2}}\right) \mid X\right)\left(\frac{y}{\sqrt{2}},-\frac{\vec{\eta}_{1}}{\sqrt{2}}\right)
\end{aligned}
$$

for s-a.e. $y \in C_{a, b}[0, T]$.

Proof. Again, as noted in the proof of Theorem 3.5, we only need to consider the case where $\lambda>0$. But using equations (4.3) and (4.4), we observe that for all $\lambda>0$,

$$
\begin{aligned}
& T_{\lambda}\left(\left[(F * G)_{\lambda} \mid X\right]\left(\cdot, \vec{\eta}_{1}\right) \mid X\right)\left(y, \vec{\eta}_{2}\right) \\
= & E_{x_{1}}\left[\left[(F * G)_{\lambda} \mid X\right]\left(y+\lambda^{-1 / 2} x_{1}-\sum_{j=1}^{n}\left(g_{j}, \lambda^{-1 / 2} x_{1}\right)^{\sim} g_{j}+\sum_{j=1}^{n} \eta_{2 j} g_{j}, \vec{\eta}_{1}\right)\right] \\
= & E_{x_{1}}\left[E _ { x _ { 2 } } \left[F \left(\frac{y}{\sqrt{2}}+\frac{1}{\sqrt{2 \lambda}}\left[x_{1}-\sum_{j=1}^{n}\left(g_{j}, x\right)^{\sim} g_{j}+x_{2}-\sum_{j=1}^{n}\left(g_{j}, x_{2}\right)^{\sim} g_{j}\right]\right.\right.\right. \\
& \left.+\sum_{j=1}^{n} \frac{\eta_{2 j}+\eta_{1 j}}{\sqrt{2}} g_{j}\right) \\
\times G\left(\frac{y}{\sqrt{2}}+\frac{1}{\sqrt{2 \lambda}}\left[x_{1}-\sum_{j=1}^{n}\left(g_{j}, x_{1}\right)^{\sim} g_{j}-x_{2}+\sum_{j=1}^{n}\left(g_{j}, x_{2}\right)^{\sim} g_{j}\right]\right. & \left.\left.\left.+\sum_{j=1}^{n} \frac{\eta_{2 j}-\eta_{1 j}}{\sqrt{2}} g_{j}\right)\right]\right] .
\end{aligned}
$$


By Lemma 4.4 above, $2^{-1 / 2}\left(x_{1}-\sum_{j=1}^{n}\left(g_{j}, x_{1}\right)^{\sim} g_{j}+x_{2}-\sum_{j=1}^{n}\left(g_{j}, x_{2}\right)^{\sim} g_{j}\right)$ and $2^{-1 / 2}\left(x_{1}-\sum_{j=1}^{n}\left(g_{j}, x_{1}\right) \sim g_{j}-x_{2}+\sum_{j=1}^{n}\left(g_{j}, x_{2}\right) \sim g_{j}\right)$ are independent processes. Hence the expectation $F G$ equals the product of the expectations and so we see that

$$
\begin{aligned}
& T_{\lambda}\left(\left[(F * G)_{\lambda} \mid X\right]\left(\cdot, \vec{\eta}_{1}\right) \mid X\right)\left(y, \vec{\eta}_{2}\right) \\
& =E_{x_{1}}\left[E _ { x _ { 2 } } \left[F \left(\frac{y}{\sqrt{2}}+\frac{x_{1}}{\sqrt{2 \lambda}}-\sum_{j=1}^{n} \frac{\left(g_{j}, x_{1}\right)^{\sim}}{\sqrt{2 \lambda}} g_{j}+\sum_{j=1}^{n} \frac{\eta_{1 j}}{\sqrt{2}} g_{j}\right.\right.\right. \\
& \left.\left.\left.+\frac{x_{2}}{\sqrt{2 \lambda}}-\sum_{j=1}^{n} \frac{\left(g_{j}, x_{2}\right)^{\sim}}{\sqrt{2 \lambda}} g_{j}+\sum_{j=1}^{n} \frac{\eta_{2 j}}{\sqrt{2}} g_{j}\right)\right]\right] \\
& \times E_{x_{1}}\left[E _ { x _ { 2 } } \left[G \left(-\left[-\left(\frac{y}{\sqrt{2}}+\frac{x_{1}}{\sqrt{2 \lambda}}-\sum_{j=1}^{n} \frac{\left(g_{j}, x_{1}\right)^{\sim}}{\sqrt{2 \lambda}} g_{j}+\sum_{j=1}^{n} \frac{\left(-\eta_{1 j}\right)}{\sqrt{2}} g_{j}\right)\right.\right.\right.\right. \\
& \left.\left.\left.\left.+\frac{x_{2}}{\sqrt{2 \lambda}}-\sum_{j=1}^{n} \frac{\left(g_{j}, x_{2}\right)^{\sim}}{\sqrt{2 \lambda}} g_{j}+\sum_{j=1}^{n} \frac{\left(-\eta_{2 j}\right)}{\sqrt{2}} g_{j}\right]\right)\right]\right] \\
& =E_{x_{1}}\left[T_{2 \lambda}(F \mid X)\left(\frac{y}{\sqrt{2}}+\frac{x_{1}}{\sqrt{2 \lambda}}-\sum_{j=1}^{n} \frac{\left(g_{j}, x_{1}\right)^{\sim}}{\sqrt{2 \lambda}} g_{j}+\sum_{j=1}^{n} \frac{\eta_{1 j}}{\sqrt{2}} g_{j}, \frac{\vec{\eta}_{2}}{\sqrt{2}}\right)\right] \\
& \times E_{x_{1}}\left[T _ { 2 \lambda } ( G ( - \cdot ) | X ) \left(-\left(\frac{y}{\sqrt{2}}+\frac{x_{1}}{\sqrt{2 \lambda}}\right.\right.\right. \\
& \left.\left.\left.-\sum_{j=1}^{n} \frac{\left(g_{j}, x_{1}\right)^{\sim}}{\sqrt{2 \lambda}} g_{j}+\sum_{j=1}^{n} \frac{\left(-\eta_{1 j}\right)}{\sqrt{2}} g_{j}\right),-\frac{\vec{\eta}_{2}}{\sqrt{2}}\right)\right] \\
& =T_{2 \lambda}\left(T_{2 \lambda}(F \mid X)\left(\cdot, \frac{\vec{\eta}_{2}}{\sqrt{2}}\right) \mid X\right)\left(\frac{y}{\sqrt{2}}, \frac{\vec{\eta}_{1}}{\sqrt{2}}\right) \\
& \times T_{2 \lambda}\left(T_{2 \lambda}(G(-\cdot) \mid X)\left(-\cdot,-\frac{\vec{\eta}_{2}}{\sqrt{2}}\right) \mid X\right)\left(\frac{y}{\sqrt{2}},-\frac{\vec{\eta}_{1}}{\sqrt{2}}\right)
\end{aligned}
$$

which concludes the proof of Theorem 4.5.

Remark 4.6. Formula (4.5) is useful in that it permits one to calculate the conditional transform of the conditional convolution without actually calculating conditional convolution.

\section{Corollaries}

Our results between the GFFT and the CP, and between the CGFFT and the CCP on function space (namely, equations (3.3) and (4.5)), are different from the results in $[8,9,10,11,15]$. But our results in Sections 3 and 4 are indeed very general theorems.

In the setting of one parameter Wiener space $\left(C_{0}[0, T], \mathcal{B}\left(C_{0}[0, T]\right), m_{w}\right)$ (i.e., the case where $a(t) \equiv 0$ and $b(t)=t$ on $[0, T])$, Huffman, Park, and Skoug 
$[8,9,10,11,15]$ showed that for appropriate functionals $F, G: C_{0}[0, T] \rightarrow \mathbb{C}$,

$$
T_{q}^{(p)}\left((F * G)_{q}\right)(y)=T_{q}^{(p)}(F)(y / \sqrt{2}) T_{q}^{(p)}(G)(y / \sqrt{2})
$$

and

$$
\begin{aligned}
& T_{q}^{(p)}\left(\left[(F * G)_{q} \mid X\right]\left(\cdot, \overrightarrow{\eta_{1}}\right) \mid X\right)\left(y, \overrightarrow{\eta_{2}}\right) \\
= & T_{q}^{(p)}(F \mid X)\left(\frac{y}{\sqrt{2}}, \frac{\overrightarrow{\eta_{2}}+\overrightarrow{\eta_{1}}}{\sqrt{2}}\right) T_{q}^{(p)}(G \mid X)\left(\frac{y}{\sqrt{2}}, \frac{\overrightarrow{\eta_{2}}-\overrightarrow{\eta_{1}}}{\sqrt{2}}\right)
\end{aligned}
$$

for s-a.e. $y \in C_{0}[0, T]$, respectively.

In next two corollaries, we assume that $T_{q}^{(p)}\left((F * G)_{q}\right), T_{q}^{(p)}(F), T_{q}^{(p)}(G)$, $T_{q}^{(p)}\left(\left[(F * G)_{q} \mid X\right]\left(\cdot, \overrightarrow{\eta_{1}}\right) \mid X\right)\left(\cdot, \overrightarrow{\eta_{2}}\right), T_{q}^{(p)}(F \mid X)\left(\cdot, \frac{\overrightarrow{\eta_{2}}+\overrightarrow{\eta_{1}}}{\sqrt{2}}\right)$, and $T_{q}^{(p)}(F \mid X)\left(\cdot, \frac{\overrightarrow{\eta_{2}}-\overrightarrow{\eta_{1}}}{\sqrt{2}}\right)$ all exist.

Corollary 5.1. In Theorem 3.5, if $a(t) \equiv 0$ and $b(t)=t$, then we have equation (5.1).

Proof. If $a(t) \equiv 0$ and $b(t)=t$, then the processes $Y_{1}$ and $Y_{2}$ in Lemma 3.4 are equivalent to the standard Wiener process. Using this fact, we see that for all $\lambda>0$

$$
T_{2 \lambda}\left(T_{2 \lambda}(F)\right)(y / \sqrt{2})=T_{\lambda}(F)(y / \sqrt{2})
$$

and

$$
T_{2 \lambda}\left(T_{2 \lambda}(G(-\cdot))(-\cdot)\right)(y / \sqrt{2})=T_{\lambda}(G)(y / \sqrt{2}) .
$$

Using equation (3.4) together with equations (5.3) and (5.4), we have equation (5.1) above.

By a similar argument, we also have following corollary.

Corollary 5.2. In Theorem 4.5, if $a(t) \equiv 0$ and $b(t)=t$, then we have equation (5.2).

\section{Examples}

In this section we apply the results obtained in previous sections to various functionals on function space $C_{a, b}[0, T]$.

Formula (3.3) is useful in that it permits one to calculate $T_{q}^{(p)}\left((F * G)_{q}\right)$ without actually calculating $(F * G)_{q}$. In practice,

$$
T_{2 q}^{(p)}\left(T_{2 q}^{(p)}(F)\right) \text { and } T_{2 q}^{(p)}\left(T_{2 q}^{(p)}(G(-\cdot))(-\cdot)\right)
$$

are usually easier to calculate than are $(F * G)_{q}$ and $T_{q}^{(p)}\left((F * G)_{q}\right)$. Also, formula (4.5) can be explained by same argument.

Example 6.1. We give this example by finding $T_{q}^{(p)}\left(\left(F_{j} * F_{k}\right)_{q}\right)$ for various functionals on function space $C_{a, b}[0, T]$. For simplicity, we restrict our discussion to the case $p=1$. In view of equations (3.3) and (4.5), we need only 
compute the transforms and the conditional transforms of the various functionals $F_{j}$. The results are summarized below where the expressions for

$$
T_{q}^{(1)}\left(T_{q}^{(1)}\left(F_{j}\right)\right), T_{q}^{(1)}\left(T_{q}^{(1)}\left(F_{j}(-\cdot)\right)(-\cdot)\right), T_{q}^{(1)}\left(T_{q}^{(1)}\left(F_{j} \mid X\right)\left(\cdot, \overrightarrow{\eta_{2}}\right) \mid X\right)\left(\cdot, \vec{\eta}_{1}\right),
$$

and

$$
T_{q}^{(1)}\left(T_{q}^{(1)}\left(F_{j}(-\cdot) \mid X\right)\left(-\cdot, \vec{\eta}_{2}\right) \mid X\right)\left(\cdot, \vec{\eta}_{1}\right)
$$

are valid for all $q \in \mathbb{R}-\{0\}$.

TABLE 1

\begin{tabular}{l|l|l}
\hline$F_{j}(x)$ & $T_{q}^{(1)}\left(T_{q}^{(1)}\left(F_{j}\right)\right)(y)$ & $T_{q}^{(1)}\left(T_{q}^{(1)}\left(F_{j}(-\cdot)\right)(-\cdot)\right)(y)$ \\
\hline$F_{1}(x)=1$ & 1 & 1 \\
$F_{2}(x)=(w, x)^{\sim}$ & $\begin{array}{l}(w, y)^{\sim} \\
+2(i / q)^{1 / 2}(w, a)_{C_{a, b}^{\prime}}\end{array}$ & $(w, y)^{\sim}$ \\
$F_{3}(x)$ & $\int_{0}^{T} x(t) d b(t)$ \\
$F_{4}(x)$ & $\begin{array}{l}\int_{0}^{T} y(t) d b(t) \\
+2(i / q)^{1 / 2} \int_{0}^{T} a(t) d b(t)\end{array}$ & $\int_{0}^{T} y(t) d b(t)$ \\
$=\exp \left\{c(w, x)^{\sim}\right\}$ & $\begin{array}{l}\exp \left\{c(w, y)^{\sim}+\frac{i c^{2}}{q}\|w\|_{C_{a, b}^{\prime}}^{2}\right. \\
\times \exp \left\{2 c(i / q)^{1 / 2}(w, a)_{C_{a, b}^{\prime}}\right\}\end{array}$ & $\exp \left\{c(w, y)^{\sim}+\frac{i c^{2}}{q}\|w\|_{C_{a, b}^{\prime}}^{2}\right\}$ \\
\hline
\end{tabular}

In Tables 1,2 and $3, w$ is any function in $C_{a, b}^{\prime}[0, T]$ and $c \in \mathbb{C}$. For all $\gamma \in \mathbb{C}$, $\gamma^{1 / 2}$ is chosen to have nonnegative real part.

Now, using Tables 1, 2, and 3, together with (3.3) and (4.5), one can immediately compute

$$
T_{q}^{(1)}\left(\left(F_{j} * F_{k}\right)_{q}\right) \text { and } T_{q}^{(1)}\left(\left[\left(F_{j} * F_{k}\right)_{q} \mid X\right]\left(\cdot, \overrightarrow{\eta_{1}}\right) \mid X\right)\left(\cdot, \overrightarrow{\eta_{2}}\right)
$$

for $j, k \in\{1,2,3,4\}$. For example,

$$
\begin{aligned}
& T_{q}^{(1)}\left(\left(F_{2} * F_{4}\right)_{q}\right)(y) \\
= & {\left[(w, y)^{\sim}+2\left(\frac{i}{2 q}\right)^{1 / 2}(w, a)_{C_{a, b}^{\prime}}\right] \exp \left\{c(w, y)^{\sim}+\frac{i c^{2}}{2 q}\|w\|_{C_{a, b}^{\prime}}^{2}\right\} }
\end{aligned}
$$


TABLE 2

\begin{tabular}{|c|c|}
\hline$F_{j}(x)$ & $T_{q}^{(1)}\left(T_{q}^{(1)}\left(F_{j} \mid X\right)\left(\cdot, \overrightarrow{\eta_{2}}\right) \mid X\right)\left(y, \overrightarrow{\eta_{1}}\right)$ \\
\hline$F_{1}(x)=1$ & 1 \\
\hline$F_{2}(x)=(w, x)^{\sim}$ & $\begin{array}{l}(w, y)^{\sim}+\sum_{j=1}^{n}\left(\eta_{2 j}+\eta_{1 j}\right)\left(w, g_{j}\right)_{C_{a, b}^{\prime}}+2(i / q)^{1 / 2}(w, a)_{C_{a, b}^{\prime}} \\
-2(i / q)^{1 / 2} \sum_{j=1}^{n}\left(w, g_{j}\right)_{C_{a, b}^{\prime}}(w, a)_{C_{a, b}^{\prime}}\end{array}$ \\
\hline$F_{3}(x)=\int_{0}^{T} x(t) d b(t)$ & $\begin{array}{l}\int_{0}^{T} y(t) d b(t)+\sum_{j=1}^{n}\left(\eta_{2 j}+\eta_{1 j}\right) \int_{0}^{T} g_{j}(t) d b(t) \\
+2(i / q)^{1 / 2} \int_{0}^{T} a(t) d b(t) \\
-2(i / q)^{1 / 2} \sum_{j=1}^{n}\left(a, g_{j}\right)_{C_{a, b}^{\prime}} \int_{0}^{T} g_{j}(t) d b(t)\end{array}$ \\
\hline$F_{4}(x)=\exp \left\{c(w, x)^{\sim}\right\}$ & $\begin{array}{l}\exp \left\{c(w, y)^{\sim}+c \sum_{j=1}^{n}\left(\eta_{2 j}+\eta_{1 j}\right)\left(w, g_{j}\right)_{C_{a, b}^{\prime}}\right. \\
\times \exp \left\{\frac{i c^{2}}{q}\|w\|_{C_{a, b}^{\prime}}^{2}-\frac{i c^{2}}{q} \sum_{j=1}^{n}\left(w, g_{j}\right)_{C_{a, b}^{\prime}}^{2}\right\} \\
\times \exp \left\{2 c(i / q)^{1 / 2}(w, a)_{C_{a, b}^{\prime}}\right\} \\
\times \exp \left\{-2 c(i / q)^{1 / 2} \sum_{j=1}^{n}\left(w, g_{j}\right)_{C_{a, b}^{\prime}}\left(g_{j}, a\right)_{C_{a, b}^{\prime}}\right\}\end{array}$ \\
\hline
\end{tabular}

and

$$
\begin{aligned}
& T_{q}^{(1)}\left(\left[\left(F_{2} * F_{4}\right)_{q} \mid X\right]\left(\cdot, \overrightarrow{\eta_{1}}\right) \mid X\right)\left(y, \overrightarrow{\eta_{2}}\right) \\
& =\left[(w, y)^{\sim}+\sum_{j=1}^{n} \frac{\eta_{2 j}+\eta_{1 j}}{\sqrt{2}}\left(w, g_{j}\right)_{C_{a, b}^{\prime}}\right. \\
& \left.\quad-2\left(\frac{i}{2 q}\right)^{1 / 2} \sum_{j=1}^{n}\left(w, g_{j}\right)_{C_{a, b}^{\prime}}(w, a)_{C_{a, b}^{\prime}}+2\left(\frac{i}{2 q}\right)^{1 / 2}(w, a)_{C_{a, b}^{\prime}}\right] \\
& \quad \times \exp \left\{c(w, y)^{\sim}+c \sum_{j=1}^{n} \frac{\eta_{2 j}-\eta_{1 j}}{\sqrt{2}}\left(w, g_{j}\right)_{C_{a, b}^{\prime}}+\frac{i c^{2}}{2 q}\|w\|_{C_{a, b}^{\prime}}^{2}\right. \\
& \left.\quad-\frac{i c^{2}}{2 q} \sum_{j=1}^{n}\left(w, g_{j}\right)_{C_{a, b}^{\prime}}^{2}\right\} .
\end{aligned}
$$

We finish this paper by mentioning that the hypotheses (and hence conclusions) of Theorems 3.5 and 4.5 above are indeed satisfied by several large classes of functionals; we shall very briefly discuss two such classes. 
TABLE 3

\begin{tabular}{l|l}
\hline$F_{j}(x)$ & $T_{q}^{(1)}\left(T_{q}^{(1)}\left(F_{2}(-\cdot) \mid X\right)\left(-\cdot, \overrightarrow{\eta_{2}}\right)\right)\left(y, \vec{\eta}_{1}\right)$ \\
\hline$F_{1}(x)=1$ & 1 \\
$F_{2}(x)=(w, x)^{\sim}$ & $(w, y)^{\sim}-\sum_{j=1}^{n}\left(\eta_{2 j}-\eta_{1 j}\right)\left(w, g_{j}\right)_{C_{a, b}^{\prime}}$ \\
$F_{3}(x)=\int_{0}^{T} x(t) d b(t)$ & $\int_{0}^{T} y(t) d b(t)-\sum_{j=1}^{n}\left(\eta_{2 j}-\eta_{1 j}\right) \int_{0}^{T} g_{j}(t) d b(t)$ \\
$F_{4}(x)=\exp \left\{c(w, x)^{\sim}\right\}$ & $\exp \left\{c(w, y)^{\sim}-c \sum_{j=1}^{n}\left(\eta_{2 j}-\eta_{1 j}\right)\left(w, g_{j}\right)_{C_{a, b}^{\prime}}\right\}$ \\
& $\times \exp \left\{\frac{i c^{2}}{q}\|w\|_{C_{a, b}^{\prime}}^{2}-\frac{i c^{2}}{q} \sum_{j=1}^{n}\left(w, g_{j}\right)_{C_{a, b}^{\prime}}^{2}\right\}$ \\
\hline
\end{tabular}

Example 6.2. The Banach algebra $\mathcal{S}\left(L_{a, b}^{2}[0, T]\right)$, introduced by Chang and Skoug in [7], consists of functionals expressible in the form

$$
F(x)=\int_{L_{a, b}^{2}[0, T]} \exp \{i\langle u, x\rangle\} d f(u)
$$

for s-a.e. $x \in C_{a, b}[0, T]$, where the associated measure $f$ is an element of $\mathcal{M}\left(L_{a, b}^{2}[0, T]\right)$, the space of $\mathbb{C}$-valued countably additive Borel measures on $L_{a, b}^{2}[0, T]$. Since the map $D_{t}: C_{a, b}^{\prime}[0, T] \rightarrow L_{a, b}^{2}[0, T]$ given by equation $(2.2)$ is an isomorphism, expression (6.1) can be rewritten by

$$
F(x)=\int_{L_{a, b}^{2}[0, T]} \exp \left\{i\left(D_{t}^{-1} u, x\right)^{\sim}\right\} d f(u)
$$

for s-a.e. $x \in C_{a, b}[0, T]$. Now for fixed $q \in \mathbb{R}$, let $\mathfrak{L}(q)$ be the class of functionals $F \in \mathcal{S}\left(L_{a, b}^{2}[0, T]\right)$ satisfying the condition

$$
\int_{L_{a, b}^{2}[0, T]} \exp \left\{\frac{5(n+1)}{\sqrt{|4 q|}}\left\|D_{t}^{-1} u\right\|_{C_{a, b}^{\prime}}\|a\|_{C_{a, b}^{\prime}}\right\}|d f(u)|<+\infty .
$$

For example for $F \in \mathfrak{L}(q)$, a direct calculation show that for all $p \in[1,2]$

$$
\begin{aligned}
& T_{2 q}^{(p)}(F \mid X)(y, \vec{\eta}) \\
= & \int_{L_{a, b}^{2}[0, T]} \exp \left\{i\langle u, y\rangle+i \sum_{j=1}^{n}\left(D_{t}^{-1} u, g_{j}\right)_{C_{a, b}^{\prime}} \eta_{j}\right.
\end{aligned}
$$




$$
\begin{aligned}
& -\frac{i}{4 q}\left[\left\|D_{t}^{-1} u\right\|_{C_{a, b}^{\prime}}^{2}-\sum_{j=1}^{n}\left(D_{t}^{-1} u, g_{j}\right)_{C_{a, b}^{\prime}}^{2}\right] \\
& \left.+i(-2 i q)^{-1 / 2}\left[\left(D_{t}^{-1} u, a\right)_{C_{a, b}^{\prime}}-\sum_{j=1}^{n}\left(D_{t}^{-1} u, g_{j}\right)_{C_{a, b}^{\prime}}\left(D_{t}^{-1} u, a\right)_{C_{a, b}^{\prime}}\right]\right\} d f(u)
\end{aligned}
$$

for s-a.e. $y \in C_{a, b}[0, T]$. Thus for s-a.e. $y \in C_{a, b}[0, T]$, we easily obtain that

$$
\left|T_{2 q}^{(p)}(F \mid X)(y, \vec{\eta})\right| \leq \int_{L_{a, b}^{2}[0, T]} \exp \left\{\frac{5(n+1)}{\sqrt{|4 q|}}\left\|D_{t}^{-1} u\right\|_{C_{a, b}^{\prime}}\|a\|_{C_{a, b}^{\prime}}\right\}|d f(u)|<+\infty .
$$

By carrying out the same calculations and using condition (6.2), we easily see that for $F$ and $G$ in $\mathfrak{L}(q)$

$$
\begin{gathered}
T_{q}^{(p)}(F)(y), T_{q}^{(p)}(F(-\cdot))(y), T_{q}^{(p)}\left(T_{q}^{(p)}(F)\right)(y), T_{q}^{(p)}\left(T_{q}^{(p)}(F(-\cdot))(-\cdot)\right)(y), \\
T_{q}^{(p)}(F \mid X)(y, \vec{\eta}), T_{q}^{(p)}(F(-\cdot) \mid X)(y, \vec{\eta}) \\
T_{q}^{(p)}\left(T_{q}^{(p)}(F \mid X)\left(\cdot, \vec{\eta}_{2}\right) \mid X\right)\left(y, \vec{\eta}_{1}\right),
\end{gathered}
$$

and

$$
T_{q}^{(p)}\left(T_{q}^{(p)}(F(-\cdot) \mid X)\left(-\cdot, \vec{\eta}_{2}\right) \mid X\right)\left(y, \vec{\eta}_{1}\right)
$$

all exist. Thus Theorems 3.5 and 4.5 hold for all $F$ and $G$ in $\mathfrak{L}(q)$.

Example 6.3. In [3], Chang, Choi, and Skoug obtained various integration by parts formulas involving generalized analytic Feynman integrals, $L_{1}$ GFFTs, and $L_{2}$ GFFTs for functionals of the form

$$
F(x)=f\left(\left\langle\alpha_{1}, x\right\rangle, \ldots,\left\langle\alpha_{m}, x\right\rangle\right)
$$

for s-a.e. $x \in C_{a, b}[0, T]$, where $\left\{\alpha_{1}, \ldots, \alpha_{m}\right\}$ is an orthonormal set of functions in $L_{a, b}^{2}[0, T]$. Proceeding formally we see that

$$
\begin{aligned}
T_{q}^{(2)}(F)(y)= & \left(\prod_{k=1}^{m} \frac{-i q}{2 \pi \sigma_{k}^{2}}\right)^{1 / 2} \int_{\mathbb{R}^{m}} f(\vec{u}+\langle\vec{\alpha}, y\rangle) \\
& \times \exp \left\{-\sum_{k=1}^{m} \frac{\left((-i q)^{1 / 2} u_{k}-m_{k}\right)^{2}}{2 \sigma_{k}^{2}}\right\} d \vec{u},
\end{aligned}
$$

where $m_{k}=\int_{0}^{T} \alpha_{k}(t) d a(t), \sigma_{k}^{2}=\int_{0}^{T} \alpha_{k}^{2}(t) d b(t)$ for $k=1, \ldots, m$, and $\langle\vec{\alpha}, y\rangle=$ $\left(\left\langle\alpha_{1}, y\right\rangle, \ldots,\left\langle\alpha_{m}, y\right\rangle\right)$. Thus, putting appropriate continuity and integrability conditions on $f: \mathbb{R}^{m} \rightarrow \mathbb{C}$ and various orthogonality between $\left\{D_{t}^{-1} \alpha_{1}, \ldots\right.$, $\left.D_{t}^{-1} \alpha_{m}\right\}$ and $\left\{g_{1}, \ldots, g_{n}\right\}$ in $C_{a, b}^{\prime}[0, T]$, one can show that Theorems 3.5 and 4.5 above hold for various functionals of the form (6.3). 


\section{References}

[1] M. D. Brue, A functional transform for Feynman integrals similar to the Fourier transform, Thesis, Univ. of Minnesota, Minneapolis, 1972.

[2] R. H. Cameron and D. A. Storvick, An $L_{2}$ analytic Fourier-Feynman transform, Michigan Math. J. 23 (1976), 1-30.

[3] S. J. Chang, J. G. Choi, and D. Skoug, Integration by parts formulas involving generalized Fourier-Feynman transforms on function space, Trans. Amer. Math. Soc. 355 (2003), 2925-2948.

[4] - Evaluation formulas for conditional function space integrals I, Stoch. Anal. Appl. 25 (2007), 141-168.

[5] _ Simple formulas for conditional function space integrals and applications, Integration: Mathematical Theory and Applications 1 (2008), 1-20.

[6] S. J. Chang and D. M. Chung, Conditional function space integrals with applications, Rocky Mountain J. Math. 26 (1996), 37-62.

[7] S. J. Chang and D. Skoug, Generalized Fourier-Feynman transforms and a first variation on function space, Integral Transforms Spec. Funct. 14 (2003), 375-393.

[8] T. Huffman, C. Park, and D. Skoug, Analytic Fourier-Feynman transforms and convolution, Trans. Amer. Math. Soc. 347 (1995), 661-673.

[9] Convolution and Fourier-Feynman transforms of functionals involving multiple integrals, Michigan Math. J. 43 (1996), 247-261.

[10] Convolution and Fourier-Feynman transforms, Rocky Mountain J. Math. 27 (1997), 827-841.

[11] Generalized transforms and convolutions, Internat. J. Math. Math. Sci. 20 (1997), 19-32.

[12] G. W. Johnson and D. L. Skoug, An $L_{p}$ analytic Fourier-Feynman transform, Michigan Math. J. 26 (1979), 103-127.

[13] H.-H. Kuo, Gaussian Measures in Banach Spaces, Lecture Notes in Math., no. 463, Springer, Berlin, 1975.

[14] C. Park and D. Skoug, A simple formula for conditional Wiener integrals with applications, Pacific J. Math. 135 (1988), 381-394.

[15] Conditional Fourier-Feynman transforms and conditional convolution products, J. Korean Math. Soc. 38 (2001), 61-76.

[16] J. Yeh, Singularity of Gaussian measures on function spaces induced by Brownian motion processes with non-stationary increment, Illinois J. Math. 15 (1971), 37-46.

[17] _ Stochastic Processes and the Wiener Integral, Marcel Dekker, Inc., New York, 1973.

Seung Jun Chang

Department of Mathematics

DANKOOK UNIVERSITY

Cheonan 330-714, Korea

E-mail address: sejchang@dankook.ac.kr

JAE Gil Choi

Department of Mathematics

DANKOOK UNIVERSITY

Cheonan 330-714, Korea

E-mail address: jgchoi@dankook.ac.kr 